\title{
Aspiración de cuerpo extraño
}

\section{Foreign body aspiration}

Andrés Benincore-Robledo, ${ }^{1}$ Gabriel Gutiérrez-Morales, ${ }^{2}$ Francisco Javier Cuevas-Schacht ${ }^{3}$

\section{INTRODUCCIÓN}

La aspiración de un cuerpo extraño es una entidad clínica que, por sus consecuencias, todo el personal de salud que tiene contacto con pacientes pediátricos debe reconocer oportunamente porque el diagnóstico correcto y la referencia oportuna con el especialista evitará las complicaciones potencialmente fatales.

\section{ANTECEDENTES HISTÓRICOS}

La aspiración de un cuerpo extraño tiene sus primeros registros en el primer decenio del año 1600; sin embargo, no fue sino hasta finales del siglo XIX cuando se reportó por primera vez la extracción de un cuerpo extraño alojado en la vía aérea. Fue Gustav Killian quien de manera exitosa logró la extracción de un hueso de cerdo alojado en el bronquio principal derecho de un adulto. ${ }^{1,2}$

A principios del decenio de 1900, en Filadelfia, Estados Unidos, Chevalier Jackson desarrolló el primer broncoscopio rígido, con lo que se revolucionó el diagnóstico y tratamiento de los cuerpos extraños alojados en la vía aérea. ${ }^{1,2}$ Este sistema, con más de 100 años de antigüedad, es la base del tratamiento que hasta el día de hoy se sigue usando.

\section{EPIDEMIOLOGÍA}

Se estima que sólo en Estados Unidos los servicios de Urgencias son visitados, aproximadamente, 17,000 veces al año por niños que aspiraron un cuerpo extraño. ${ }^{3}$ Las muertes por asfixia secundaria a la aspiración de un cuerpo extraño se reportan entre 100 y 200 por año (7\% de muertes por accidentes en casa en menores de 3 años). ${ }^{4,5}$

En México se carece de estadísticas precisas al respecto; sin embargo, en tres series reportadas en nuestra población, una en el Instituto Nacional

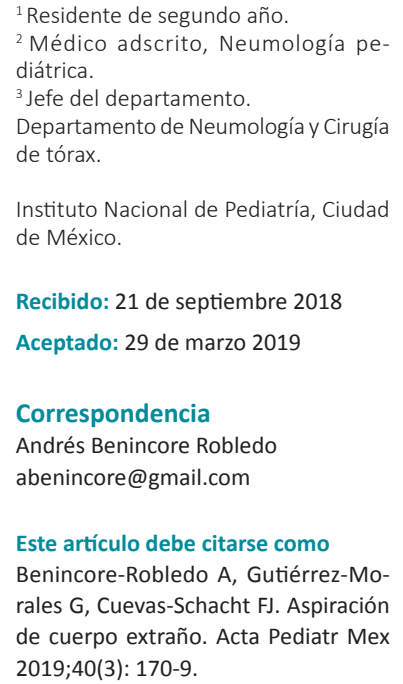

Este artículo debe citarse como Benincore-Robledo A, Gutiérrez-Morales G, Cuevas-Schacht FJ. Aspiración de cuerpo extraño. Acta Pediatr Mex 2019;40(3): 170-9. 
Benincore-Robledo A, et al. Aspiración de cuerpo extraño

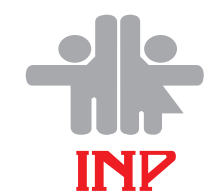

de Pediatría, en el Hospital Infantil de México y otra en el Centro Médico Nacional Siglo XXI, se estima una incidencia de 16, 22.5 y 6.6 casos al año, respectivamente. ${ }^{1,2,6}$

\section{ETIOPATOGENIA}

La edad más frecuente en la que suceden las aspiraciones de cuerpos extraños es entre 1 y 3 años (70\%). ${ }^{1,6}$ La etapa del desarrollo de los primeros años de vida del niño se caracteriza por la exploración de su entorno, de ahí que de manera refleja se lleven a la boca cualquier objeto que esté a su alcance. Esta curiosidad innata, junto con la inmadurez de los músculos faríngeos y laríngeos propia de su edad, hacen que un momento de desatención de los cuidadores derive en un evento de aspiración.

En los niños preescolares y escolares la mayor parte de los eventos de aspiración suceden durante el juego, porque a esa edad lo común es que corran, brinquen y griten mientras comen o tienen otros objetos en la boca. Es frecuente encontrar semillas, cacahuates y otros cuerpos extraños biológicos en la vía aérea, ${ }^{2,7,8}$ lo mismo que objetos plásticos y metálicos que forman parte del juego.

La incidencia de aspiración de cuerpo extraño en los adolescentes es bastante más baja que en los grupos que les anteceden. Las tapas de plástico de los bolígrafos, alfileres, clavos, clips y aretes son algunos de los objetos que suelen "detener" en la boca mientras realizan diversas tareas, y es ahí cuando de manera accidental, por un suspiro, bostezo, o asombro el objeto es aspirado.

\section{CUADRO CLÍNICO}

El cuadro inicial más frecuente es un evento súbito de tos, ahogamiento, cianosis y dificultad respiratoria., ${ }^{1,5,8,9}$ En algunas ocasiones el episodio puede ser presenciado por un adulto u otro niño; Si es así el diagnóstico será evidente. De lo contrario, el motivo de consulta será tos y dificultad respiratoria, clínica compatible con otros diagnósticos diferenciales, por lo que se requiere un alto índice de sospecha para no pasarlo por alto.

Posterior al evento agudo hay dos opciones: el cuerpo extraño, por sus características físicas y su posición en la vía aérea, causa obstrucción importante del flujo del aire; por lo tanto, los síntomas son evidentes y estrepitosos (tos, dificultad respiratoria, sibilancias, sensación de ahogo, estridor, disfonía), o el cuerpo extraño se acomoda en algún sitio donde la obstrucción es mínima y la clínica escasa (por lo general en la vía aérea distal). Esta etapa, denominada silenciosa u oligosintomática, ${ }^{1}$ puede hacer pensar a los cuidadores y personal de salud que ya no existe peligro, que el cuerpo extraño ya se expulsó o, en caso de no haber sido presenciado, que nunca existió.

Esta peligrosa suposición lleva a una tercera etapa en la que el cuerpo extraño alojado en la vía aérea ejerce efectos locales en la mucosa, ocasiona inflamación, obstrucción e infección, según sea la naturaleza del objeto, y tendrá diferentes complicaciones con diversas expresiones clínicas. Los cuadros esporádicos de tos y sibilancias pueden hacer sospechar, erróneamente, en asma o bronquiolitis. La tos con flemas persistentes, cuadros de neumonías de repetición, falla de medro, o, inclusive, cuadros de sepsis por mediastinitis secundaria a la perforación de la vía aérea por el cuerpo extraño son algunas de las otras manifestaciones que se pueden observar en esta etapa. ${ }^{1,9}$

Es decisivo que todo el personal de salud que atiende pacientes pediátricos sepa identificar adecuadamente a los niños con sospecha de aspiración de cuerpo extraño para que se pueda indicar el tratamiento correcto y oportuno. El concepto de "sospecha fundada" de aspiración de cuerpo extraño, descrito por Pérez Fernández hace casi 30 años, ${ }^{1}$ y la guía de tratamiento del 
paciente con este diagnóstico, propuesta por el mismo autor, ejemplifica de manera concisa lo que debe hacerse ante una situación como la descrita.

El concepto de "sospecha fundada" consiste en asumir que un niño en el que se tenga la mínima sospecha de que haya aspirado un cuerpo extraño, y tenga un cuadro clínico compatible, sea estudiado hasta descartar de manera inobjetable su existencia.

\section{ESTUDIOS DE IMAGEN}

La radiografía de tórax es el primer estudio en un paciente pediátrico que llega al servicio de urgencias con sospecha de aspiración de cuerpo extraño. Gran parte de los objetos aspirados son radiolúcidos (biológicos y plásticos) y, por lo tanto, no se ven por este medio. Solo se logrará observar el cuerpo extraño en 15\% de los casos (metálicos y huesos). ${ }^{1,6,8}$ Es más común identificar datos indirectos (aparecen hasta en $50 \%$ de las radiografías), que son consecuencia de la obstrucción en la vía aérea (atrapamiento de aire, atelectasias, consolidación, neumotórax) (Figuras 1-5). Respecto de las radiografías de tórax es que, incluso $45 \%$ pueden interpretarse normales, lo que puede resultar en un diagnóstico erróneo. ${ }^{1,6,9}$

La tomografía axial computada de tórax ha tomado fuerza como ayuda diagnóstica en casos de sospecha de aspiración, en los que la radiografía de tórax no es concluyente. Un estudio efectuado en Francia reportó sensibilidad de 98\% y especificidad de $94 \%$ (valor predictivo positivo $99 \%$ y valor predictivo negativo $86.5 \%)^{10}$ con este recurso con reconstrucción multiplanos. En este estudio las tomografías se interpretaron en dos ocasiones (por un radiólogo y por un ciru-
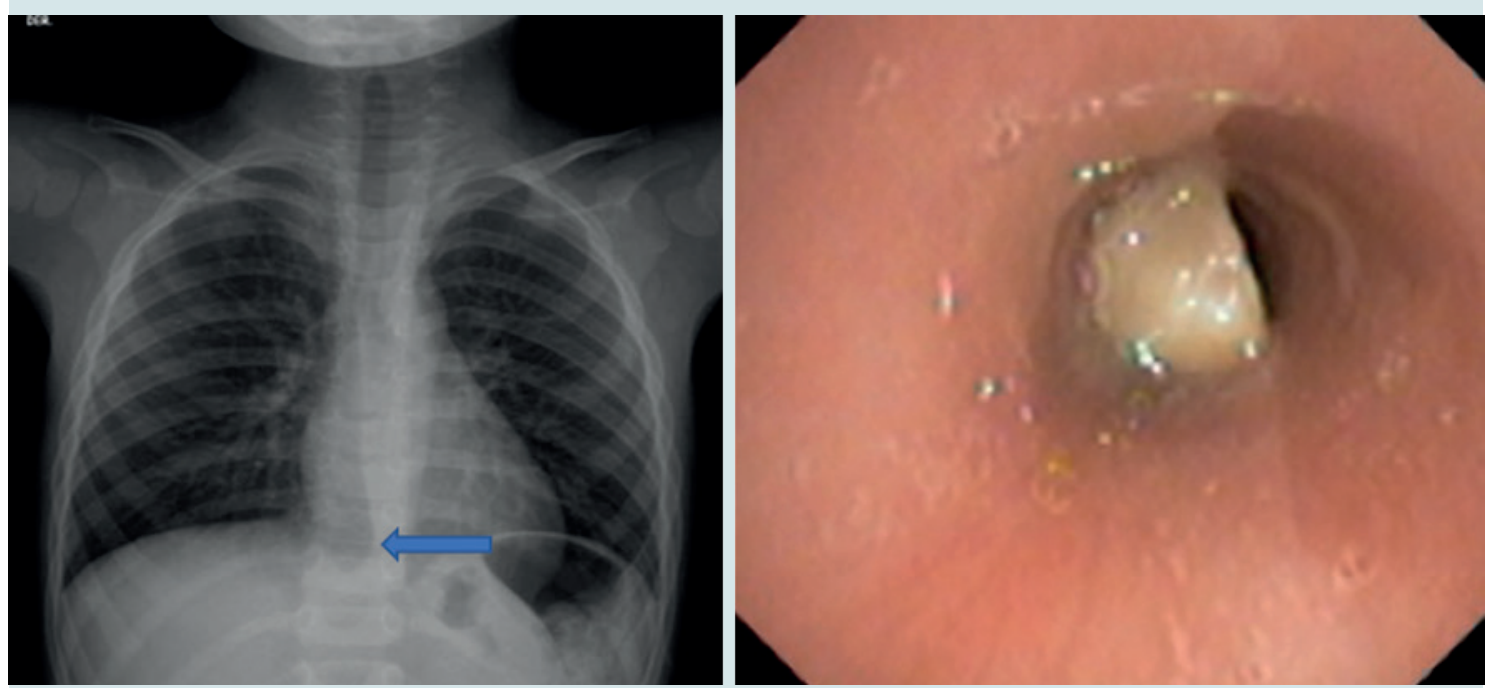

Figura 1. Paciente masculino de 2 años, 5 meses, con atragantamiento y cianosis mientras comía cacahuates y jugaba. En el hospital observaron que la radiografía de tórax no mostraba alguna anormalidad, por eso lo dieron de alta, solo con ibuprofeno y salbutamol. Ante la persistencia de la tos otra radiografía de tórax mostró sobredistención pulmonar bilateral, herniación del parénquima pulmonar derecho sobre la línea media (flecha). La broncoscopia puso de manifiesto el cuerpo extraño biológico en el tercio superior de la tráquea. Se extrajo sin complicaciones. 
Benincore-Robledo A, et al. Aspiración de cuerpo extraño

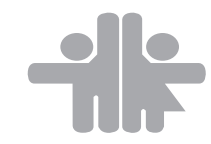

INP

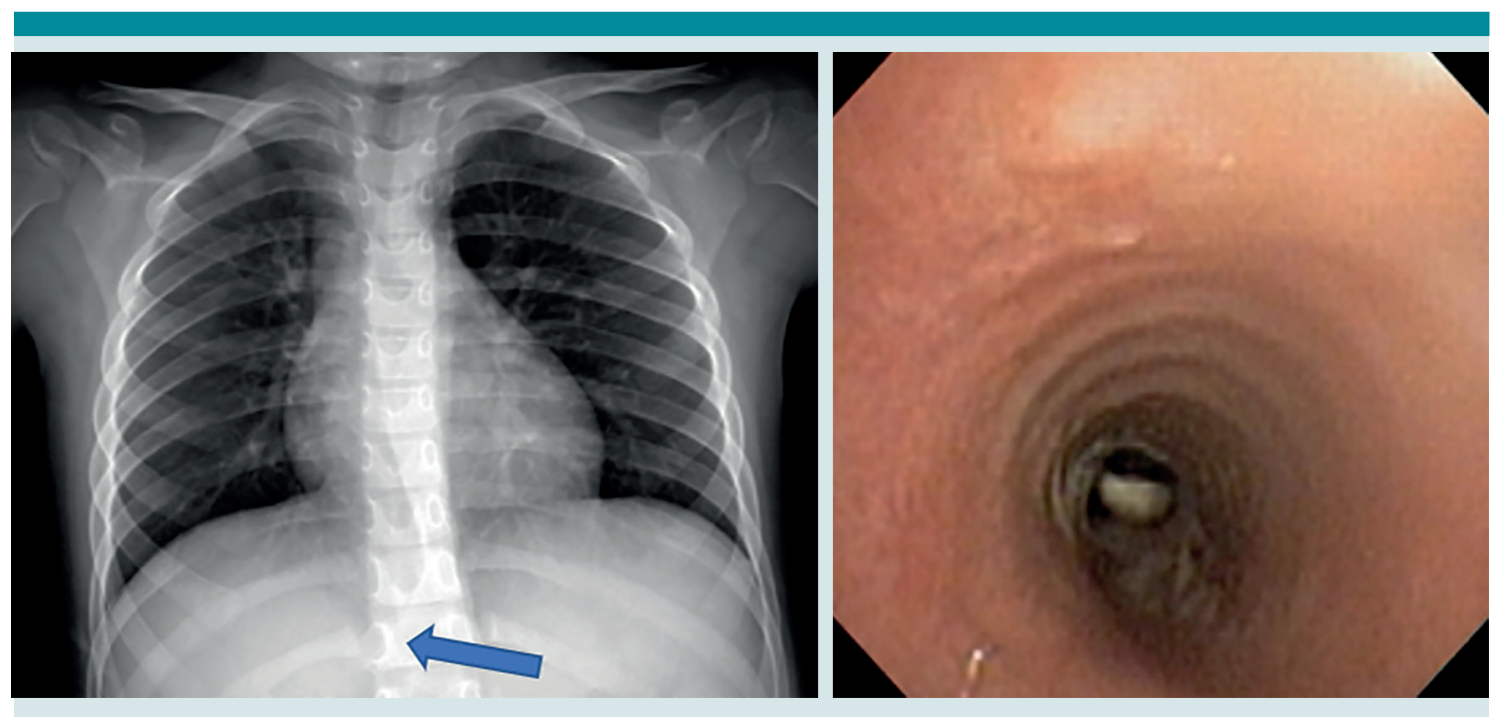

Figura 2. Paciente femenina de 4 años 11 meses. Tos y dificultad respiratoria mientras corría y comía cacahuates. Tuvo fiebre, tos y sibilancias. Fue llevada al hospital y solo le indicaron un antibiótico y salbutamol. Ante la persistencia de los síntomas por tres semanas se volvió a tomar una nueva radiografía de tórax: sobredistención pulmonar bilateral, herniación del parénquima pulmonar derecho sobre la línea media que sobrepasa el hemidiafragma (flecha)). La broncoscopia puso de manifiesto un cuerpo extraño biológico en el tercio inferior de la tráquea. Se extrajo sin complicaciones.

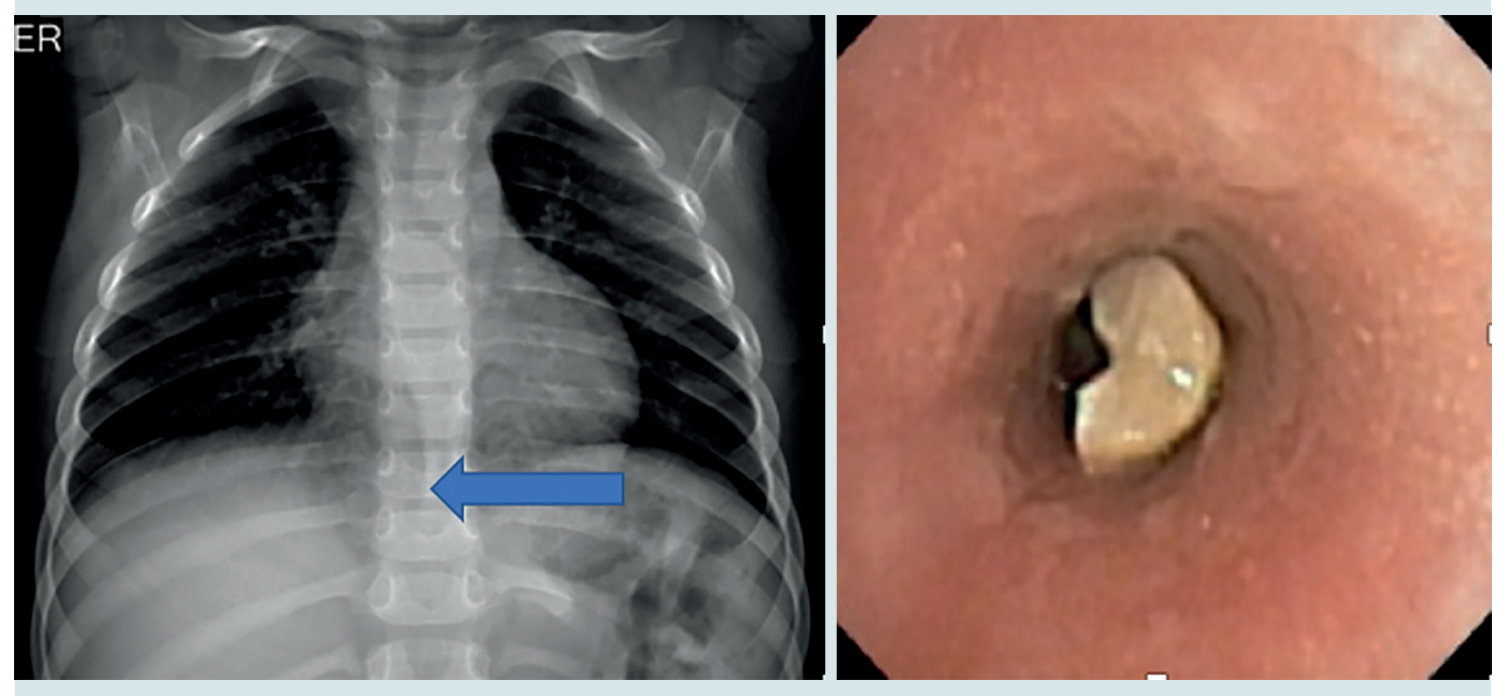

Figura 3. Paciente masculino de 2 años, con tos y cianosis mientras jugaba y comía cacahuates. En el hospital le tomaron una radiografía y lo dieron de alta con la sola indicación de un antibiótico. Ante la persistencia de la tos se le tomó una nueva radiografía de tórax que mostró sobredistención pulmonar bilateral, herniación del parénquima pulmonar derecho sobre la línea media (flecha). La broncoscopia advirtió la existencia de un cuerpo extraño biológico en el tercio superior de la tráquea. Se extrajo sin complicaciones. 


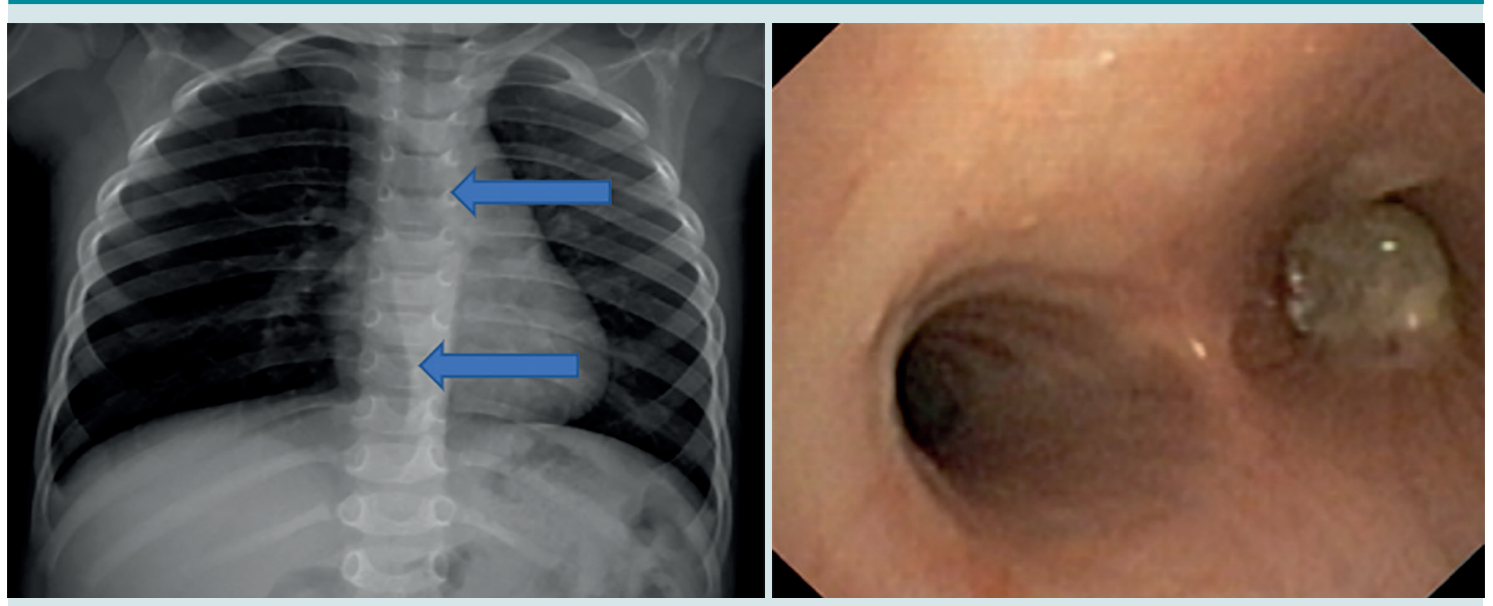

Figura 4. Paciente masculino de 2 años 6 meses con tos, dificultad respiratoria y cianosis mientras comía cacahuates y corría. La radiografía de tórax mostró: asimetría por sobredistención del hemitórax derecho, con aumento en la radiolucidez y herniación del parénquima que sobrepasaba la línea media y el hemidiafragma (flechas). La broncoscopia advirtió la existencia de un cuerpo extraño biológico en el bronquio principal derecho. Se extrajo sin complicaciones.

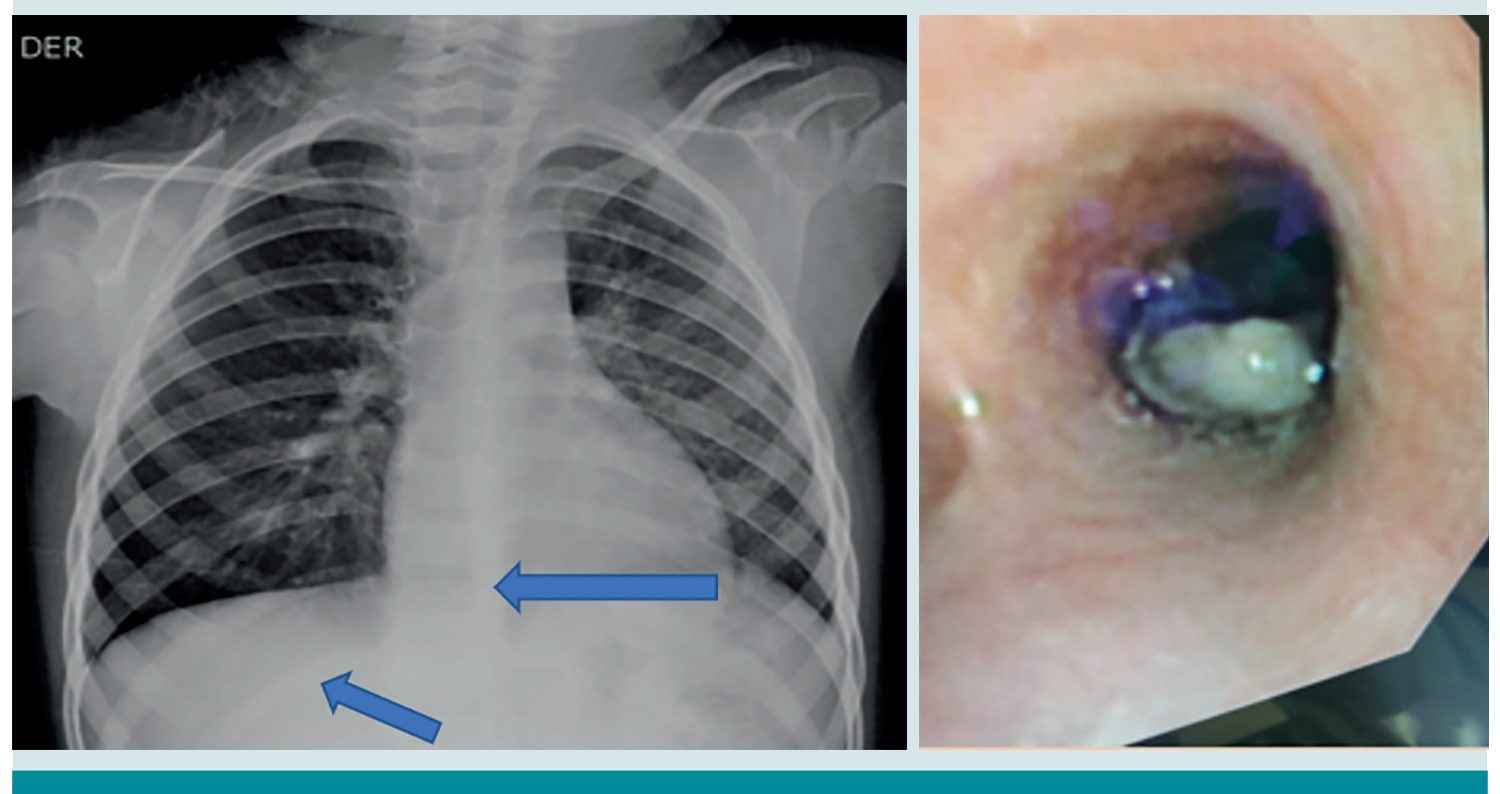

Figura 5. Paciente femenina de 3 años 9 meses. Mientras jugaba tuvo un cuadro de tos súbita, dificultad respiratoria y cianosis. En el Centro de Salud le administraron salbutamol por sibilancias; la dieron de alta. Permaneció cuatro meses con tos, fiebre, sibilancias y múltiples tratamientos para el asma y neumonía. La radiografía de tórax mostró sobredistención en el hemitórax derecho, herniación del parénquima que sobrepasaba la línea media y el hemidiafragma (flechas). La broncoscopia mostró un cuerpo extraño biológico en el bronquio principal derecho. Se extrajo sin complicaciones. 
jano de tórax). Al ser la interpretación de la TAC dependiente de la experiencia del observador, es posible que esos resultados no puedan extrapolarse a hospitales donde la interpretación la hace el médico de primer contacto. Si a esto se suma el alto costo, poca accesibilidad en centros de primer y segundo nivel, y la necesidad de sedación en ciertos pacientes (lactantes, preescolares principalmente), puede concluirse que la tomografía axial computada de tórax no es el estudio diagnóstico de elección en nuestro medio.

\section{BRONCOSCOPIA}

La broncoscopia, desde su invención a principios del siglo $X X$, ha sido la principal herramienta diagnóstica y terapéutica para los casos de aspiración de cuerpo extraño. Permite la visualización directa de la vía aérea desde las coanas hasta la tercera o cuarta subdivisión bronquial (según la edad del paciente), ${ }^{11}$ por lo que es la manera más certera de confirmar o descartar la existencia de un cuerpo extraño.

La broncoscopia rígida ha sido el método de elección para la extracción de cuerpos extraños alojados en la tráquea o los bronquios principales. Su amplio canal de trabajo permite la ventilación del paciente durante el procedimiento, con una visualización adecuada e instrumentación accesible. ${ }^{2,11}$ En los últimos años y con el advenimiento de mejores instrumentos, la broncoscopia flexible ha ganado terreno no sólo en el diagnóstico, sino también en el tratamiento de la aspiración de un cuerpo extraño. La posibilidad de llegar a segmentos más distales dentro del árbol traqueo-bronquial, la mayor facilidad para maniobrar dentro de éste, así como la menor cantidad de lesiones en la mucosa que causa el equipo flexible son algunas de las ventajas que han llevado a varios grupos a preferir este procedimiento en lugar del rígido. ${ }^{11,12}$

La decisión de qué tipo de broncoscopio usar será, finalmente, del médico, con base en su experiencia, características del cuerpo extraño, y disponibilidad del equipo.

En raras ocasiones el cuerpo extraño no podrá extraerse con el broncoscopio (menos de $2 \%$ de los casos), en cuyo caso será necesario un procedimiento quirúrgico de urgencia (toracotomía y broncotomía) para su extracción. ${ }^{13}$

\section{TRATAMIENTO}

En ocasiones, los cuidadores que se enfrentan a casos de niños con aspiración de cuerpo extraño, por pánico o ignorancia, pueden llevar a cabo algunas acciones que por sí mismas causan daño, incluso mayor que el del propio cuerpo extraño. Introducir el dedo a ciegas en la boca del niño (Figura 6), provocarle vómito, darle palmadas en la espalda, poner al niño de cabeza, sacudir, o dar a comer diversos alimentos (plátano, huevo crudo, aceites) ${ }^{1}$ puede ocasionar que un objeto extraño que se encontraba en la orofaringe o en la boca se impacte en la vía aérea, que al provocar vómito haya bronco-aspiración, o que un cuerpo extraño que estaba alojado en un bronquio principal y que sólo ocasionaba obstrucción en un pulmón, migre a la tráquea y obstruya la totalidad del flujo de aire.

Cualquier paciente con un episodio súbito de tos, dificultad respiratoria o atragantamiento, o cualquiera de los signos y síntomas descritos en el Cuadro 1, entra en el concepto de "sospecha fundada" de aspiración de cuerpo extraño. El fin de este concepto es asegurar que un niño con cualquier dato clínico compatible sea estudiado, ya sea hasta la extracción del cuerpo extraño, o hasta descartar de manera irrefutable la existencia de éste.

Cuando el paciente llega al servicio de urgencias en la etapa aguda con datos clínicos de insuficiencia respiratoria, lo primero es estabilizarlo siguiendo el "C-A-B" propuesto en el programa 

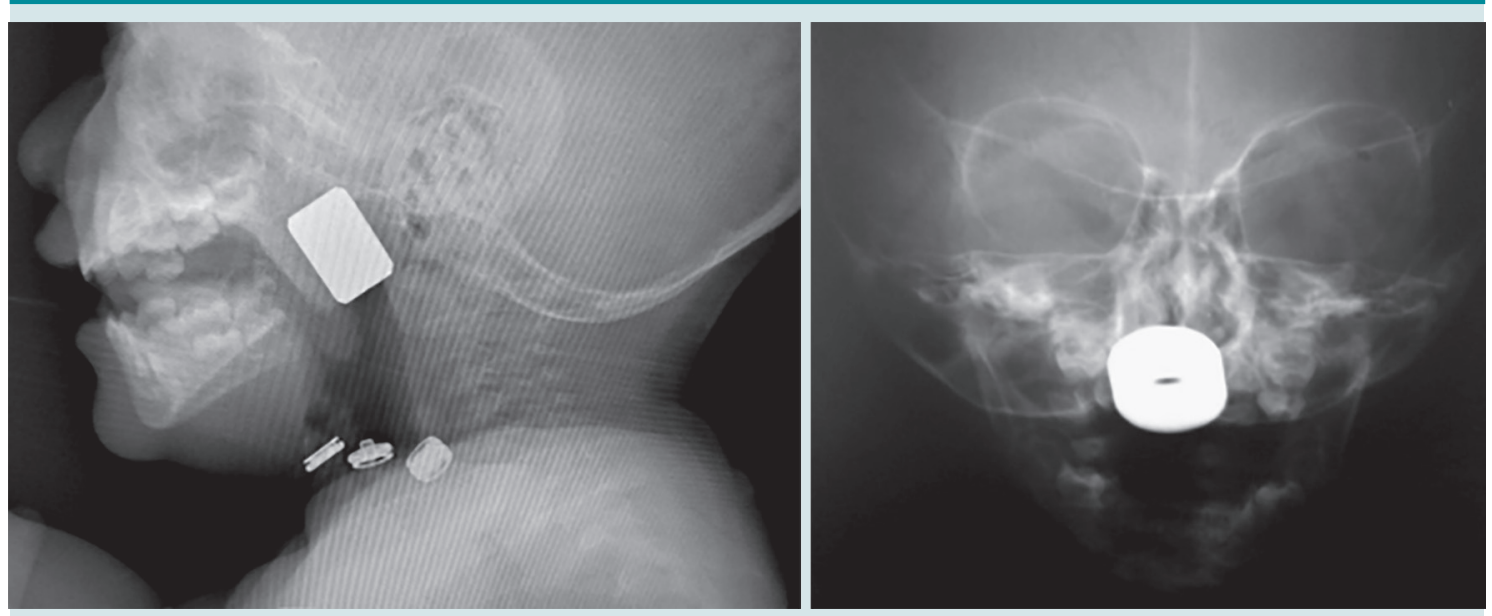

Figura 6. Paciente masculino de 2 años, con un cuerpo extraño metálico empujado por la madre hacia la nasofaringe al intentar sacarlo con el dedo. INP 2018.

Cuadro 1. Manifestaciones clínicas de la aspiración de un cuerpo extraño

\begin{tabular}{lcc}
\hline Etapa aguda & Etapa oligosintomática & Etapa crónica \\
\hline Crisis de tos & Puede haber tos leve, & Tos crónica \\
\hline Dificultad respiratoria & sibilancias aisladas o & Sibilancias/Asma \\
\hline Sibilancias & sensación de cuerpo extraño. & Disnea de esfuerzos \\
\hline Estridor & Las manifestaciones clínicas & Neumonías de repetición \\
\hline Disfonía & pueden no ser suficientemente severas & Fiebre sin foco/sepsis \\
Cianosis & como para preocupar a & Supuración broncopulmonar \\
\hline Sensación de ahogo & cuidadores o personal de salud. &
\end{tabular}

Modificada de: Pérez-Fernández L.F. Toma de decisiones para diagnóstico y tratamiento del niño que probablemente deglutió o aspiró un cuerpo extraño. En: Pérez-Fernández L.F. Neumología y Cirugía de Tórax. Toma de decisiones para diagnóstico y tratamiento. 2001.

pediátrico de Soporte Vital Avanzado Pediátrico de la Asociación Americana del Corazón (PALS/ AHA-Pediatric Advanced Life Support/American Heart Association), ${ }^{14}$ y realizar un estudio endoscópico urgente de la vía aérea.

Si al momento de consultar el paciente se encuentra en la etapa aguda, pero está estable, es decir, sin datos clínicos de inestabilidad cardiorrespiratoria, o se encuentra en la etapa oligosintomática pueden solicitarse los estudios de imagen. La radiografía de tórax es el principal recurso con el que cuentan la mayor parte de los servicios de urgencias. Es rápido, fácil, poco costoso y no requiere sedación. En caso de que sea un objeto radio-opaco, aportará el diagnóstico de manera rápida, y dará la localización del objeto (Figura 7). En caso de ser radiolúcido, la radiografía puede ser normal o mostrar los datos indirectos de obstrucción comentados. La 


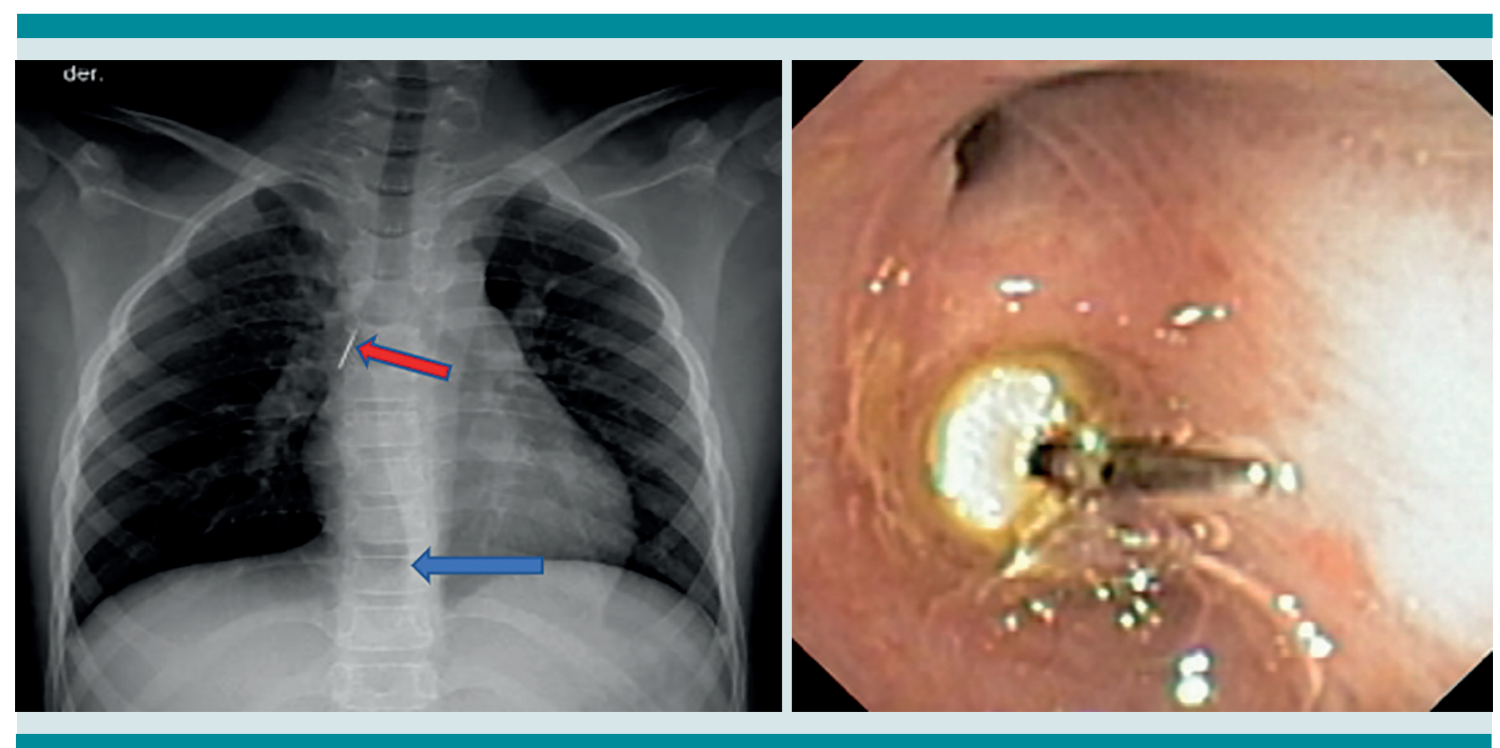

Figura 7. Paciente masculino de 12 años que súbitamente tuvo un acceso de tos y dificultad respiratoria luego de "tragarse" una tachuela que sostenía en la boca. La radiografía de tórax mostró herniación del parénquima pulmonar derecho que sobrepasaba la línea media (flecha azul). Imagen opaca (densidad metal) en la región del bronquio principal derecho (flecha roja). La broncoscopia mostró un cuerpo extraño metálico en el bronquio principal derecho. Se extrajo sin complicaciones.

tomografía axial computada de tórax puede considerarse si se cuenta con el recurso, tomando en cuenta lo mencionado.

Sin importar el resultado de los estudios de imagen, la broncoscopia debe estar en la mente del médico desde el ingreso del paciente, y ninguna de estas intervenciones debe retrasar el estudio endoscópico.

El esquema de toma de decisiones se resume en la Figura 8.

\section{CONCLUSIONES}

La aspiración de un cuerpo extraño es un evento accidental que ocurre con mayor frecuencia en niños de 1-3 años. Cualquier niño con un cuadro clínico de tos de inicio súbito, dificultad respiratoria o ahogamiento debe considerarse con "sospecha fundada de aspiración de cuerpo extraño", aunque no lo hayan presenciado sus cuidadores.

Un paciente con antecedentes sugerentes de aspiración, aunque se encuentre asintomático al momento de la consulta, debe revisarse con el mismo concepto de "sospecha fundada de aspiración de cuerpo extraño". La fase oligosintomática puede hacer pasar por alto el diagnóstico si no se tiene un alto índice de sospecha. Los estudios de imagen son útiles para confirmar la existencia de un cuerpo extraño, pero nunca para descartarlo. Los cuerpos extraños biológicos (los más frecuentemente aspirados) son radiolúcidos y no se ven en la radiografía.

Sin importar el reporte de los estudios de imagen, un niño con "sospecha fundada de aspiración de cuerpo extraño" debe ser explorado con en- 


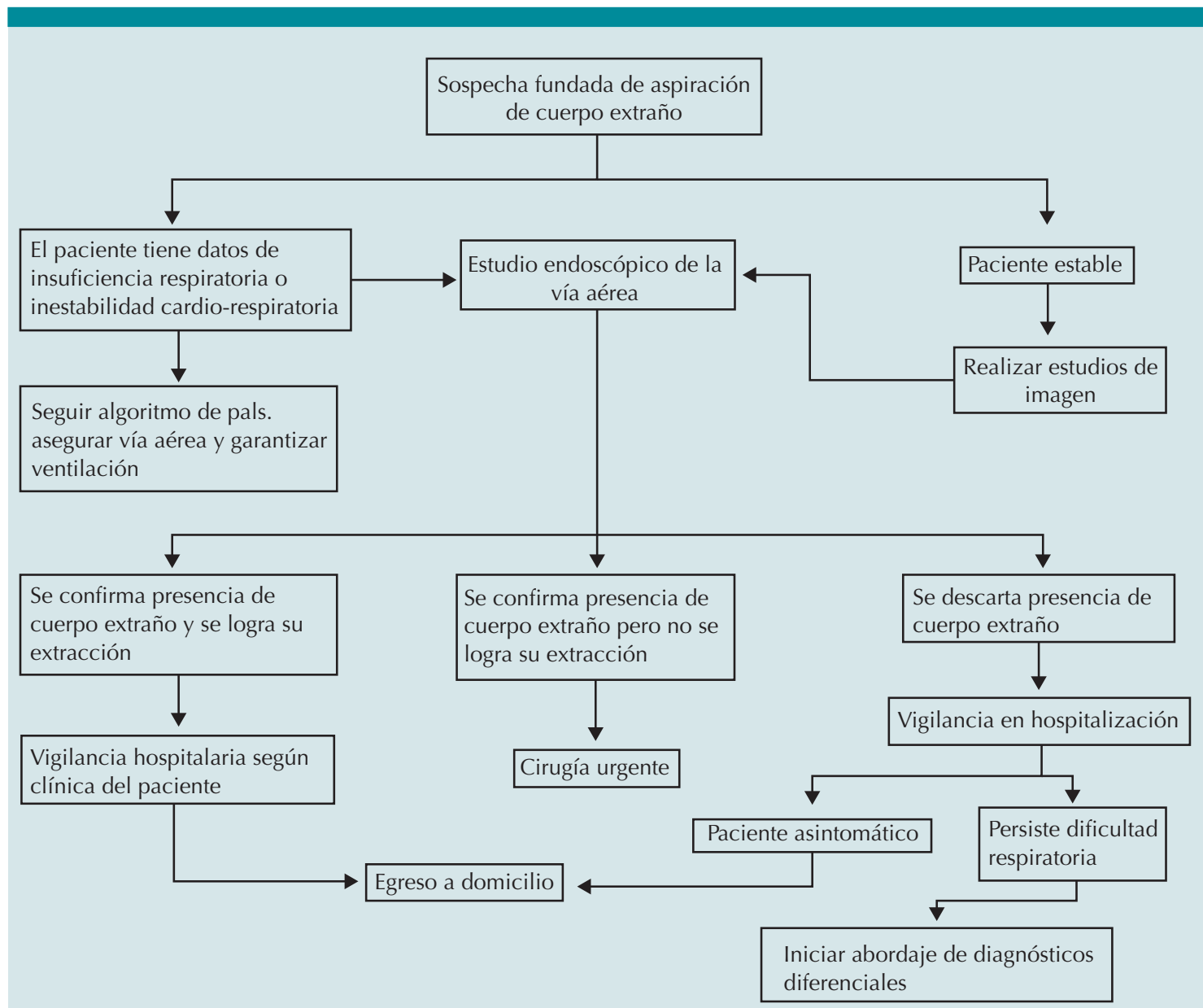

Modificada de: Pérez-Fernández L.F. Toma de decisiones para diagnóstico y tratamiento del niño que probablemente deglutió o aspiró un cuerpo extraño. En: Pérez-Fernández L.F. Neumología y Cirugía de Tórax. Toma de decisiones para diagnóstico y tratamiento. 2001.

Figura 8.

doscopia de la vía aérea. Nunca debe retrasarse su práctica mientras se espera un estudio de imagen. Figura 8

\section{REFERENCIAS}

1. Pérez-Fernandez L. Deglución o aspiración de cuerpos extraños. Criterios Pediatr. 1991;7(c):89-92. doi:10.15713/ ins.mmj.3.
2. Ramírez-Figueroa JL, et al. Foreign body removal by flexible fiberoptic bronchoscopy in infants and children. Pediatr Pulmonol. 2005;40(5):392-97. doi:10.1002/ppul.20242.

3. Kim IA, Shapiro N, Bhattacharyya N. The national cost burden of bronchial foreign body aspiration in children. Laryngoscope. 2015;125(5):1221-24. doi:10.1002/lary.25002.

4. Brkic F, et al. Death as a Consequence of foreign body aspiration in children. Med Arch. 2018;72(3):220. doi:10.5455/ medarh.2018.72.220-223.

5. Sheehan CC, Lopez J, Elmaraghy CA. Low rate of positive bronchoscopy for suspected foreign body aspiration in 
Benincore-Robledo A, et al. Aspiración de cuerpo extraño

infants. Int J Pediatr Otorhinolaryngol. 2018;104(September):72-75. doi:10.1016/j.ijporl.2017.10.030.

6. Aguirre Vázquez IR. Aspiración de cuerpos extraños en pediatría. Experiencia de 15 años. Análisis de 337 casos. Cirugía Pediátric 2013;26:4.

7. Carlos $\mathrm{L}$, et al. Diagnóstico tardío de la aspiración de un cuerpo extraño. Bol Hosp Infant Mex. 2011;68(3):220-24.

8. Baram A, et al. Tracheobronchial Foreign Bodies in Children: The Role of Emergency Rigid Bronchoscopy. Glob Pediatr Heal. 2017;4:2333794X1774366doi:10.1177/233 $3794 \times 17743663$.

9. Chen $X$, Zhang $C$. Foreign body aspiration in children: Focus on the impact of delayed treatment. Int J Pediatr Otorhinolaryngol. 2017;96:111-15. doi:10.1016/j. ijporl.2017.03.013.
10. Pitiot $\mathrm{V}$, et al. The use of CT-scan in foreign body aspiration in children: A 6 years' experience. Int J Pediatr Otorhinolaryngol. 2017;102:169-73. doi:10.1016/j.ijporl.2017.08.036.

11. Eber $E$, et al. ERS statement: interventional bronchoscopy in children. Eur Respir J. 2017;50(6):1700901. doi:10.1183/13993003.00901-2017.

12. Tenenbaum T, et al. Management of Foreign Body Removal in Children by Flexible Bronchoscopy. J Bronchol Interv Pulmonol. 2017;24(1):21-28. doi:10.1097/ LBR.0000000000000319.

13. Kiran $\mathrm{S}$, et al. Bronchotomy for removal of foreign body bronchus in an infant. Indian J Anaesth. 2014;58(6):772-3. doi: 10.4103/0019-5049.147156

14. American Academy of Pediatrics., \& American Heart Association. (2016). Pediatric advanced life support. 